\title{
Floristic composition and community structure of a seasonally deciduous forest on limestone outcrops in Central Brazil
}

\author{
JEANINE M. FELFILI ${ }^{1,3}$, ANDRÉ R. TERRA NASCIMENTO ${ }^{2}$, \\ CHRISTOPHER WILLIAM FAGG ${ }^{1}$ and ELISA MARIA MEIRELLES ${ }^{1}$
}

(received: September 20, 2004; accepted: June 18, 2007)

\begin{abstract}
Floristic composition and community structure of a seasonally deciduous forest on limestone outcrops in Central Brazil). The objective of this study was to analyze the floristic composition and structure of the tree component in a seasonally deciduous forest on limestone outcrops, located in the northeast region of Goiás State, Brazil. A sample composed of 25 randomly sampled plots of $20 \times 20 \mathrm{~m}\left(400 \mathrm{~m}^{2}\right)$ within a 50 ha forest, was measured. All woody individuals $\geq 5 \mathrm{~cm}$ diameter at breast height (DBH) had their diameter and height measured. The tree community was composed of 39 species with a density of 734 individuals ha ${ }^{-1}$; the richest families were Leguminosae (11 species), Bignoniaceae (4 species) and Apocynaceae (4 species). The forest had a basal area of $16.37 \mathrm{~m}^{2} \mathrm{ha}^{-1}$, with the most important species Cavanillesia arborea $\left(3.26 \mathrm{~m}^{2} \mathrm{ha}^{-1}\right)$, Pseudobombax tomentosum $\left(2.35 \mathrm{~m}^{2} \mathrm{ha}^{-1}\right)$, Dilodendron bipinnatum $\left(1.84 \mathrm{~m}^{2} \mathrm{ha}^{-1}\right)$, Tabebuia impetiginosa $\left(1.36 \mathrm{~m}^{2} \mathrm{ha}^{-1}\right)$ and Myracrodruon urundeuva $\left(1.26 \mathrm{~m}^{2} \mathrm{ha}^{-1}\right)$ occupying $61.5 \%$ of the total basal area of the forest. Several species grew on rocks, or in rock fissures or in places with a shallow layer of soil or even in litter over rocky layers. The floristic composition showed links with the "Caatinga" flora, with other patches of seasonal forests in Central Brazil and in the Pantanal, and with the Chiquitano forests of Bolivia too, containing even two species considered as endemic to the "Caatinga".
\end{abstract}

Key words - "Caatinga", Central Brazil, "Cerrado" biome, seasonally dry forests

RESUMO - (Composição florística e fitossociologia de uma floresta estacional decidual sobre afloramento de calcário no Brasil Central). O objetivo deste estudo foi analisar a composição de espécies lenhosas e a estrutura de um fragmento de Floresta Estacional Decidual sobre afloramento de calcário, situado na Região Nordeste de Goiás, Brasil. Foram amostradas vinte e cinco unidades amostrais quadradas de $20 \times 20 \mathrm{~m}\left(400 \mathrm{~m}^{2}\right) \mathrm{em}$ um fragmento florestal de 50 ha, sendo incluídos na amostragem todos os indivíduos com diâmetros à altura do peito (DAP) igual ou superior a $5 \mathrm{~cm}$ que tiveram seus diâmetros e alturas mensurados. A comunidade arbórea apresentou uma riqueza de 39 espécies e uma densidade de 734 indivíduos por hectare, com maior representação das famílias Leguminosae (11 espécies), Bignoniaceae (4 espécies) e Apocynaceae (4 espécies), destacando-se sobre as demais famílias botânicas. A floresta apresentou uma área basal de $16,37 \mathrm{~m}^{2} \mathrm{ha}^{-1}$, com as espécies Cavanillesia arborea $\left(3,26 \mathrm{~m}^{2} \mathrm{ha}^{-1}\right)$, Pseudobombax tomentosum $\left(2,35 \mathrm{~m}^{2} \mathrm{ha}^{-1}\right)$, Dilodendron bipinnatum $\left(1,84 \mathrm{~m}^{2} \mathrm{ha}^{-1}\right)$, Tabebuia impetiginosa $\left(1,36 \mathrm{~m}^{2} \mathrm{ha}^{-1}\right)$ e Myracrodruon urundeuva $\left(1,26 \mathrm{~m}^{2} \mathrm{ha}^{-1}\right)$ destacando-se sobre as demais, e somando em conjunto, $61,5 \%$ da área basal da floresta. Várias espécies cresceram sobre as rochas, outras nas fissuras ou em locais com solos rasos ou apenas sobre serapilheira cobrindo camadas rochosas. A composição florística mostrou afinidade com a flora da caatinga, com outras manchas de floresta estacional no Brasil Central e no Pantanal, e nas florestas Chiquitanas na Bolívia também, contendo inclusive duas espécies consideradas endêmicas da Caatinga.

Palavras-chave - Brasil Central, bioma Cerrado, Caatinga, floresta estacional decidual

\section{Introduction}

Limestone outcrops can support tropical forests worldwide, and sometimes these forests are regarded as relict communities with many endemic species (Gentry 1995, Pérez-Garcia \& Meave 2005, 2006). Chemical activity of these basic rocks is confined almost entirely

\footnotetext{
1. Universidade de Brasília, Departamento de Engenharia Florestal, Caixa Postal 04357, 70919-970 Brasília, DF, Brazil

2. Universidade Federal de Uberlândia, Instituto de Biologia, Caixa Postal 593, 38400-902 Uberlândia, MG, Brazil

3._Corresponding author: felfili@unb.br
}

to the vegetation soil interface and to the bedrock directly in contact with soil or plant roots, and comparatively little occurs within the main body of limestone (Crowther 1987).

In China, the species diversity of monsoon forests on limestone outcrops was low compared to several other forest types studied. According to Cao \& Zhang (1997) this presumably results from its extremely harsh microenvironment where a great number of large limestone rocks come up out of the soil and cover the ground. This, in addition to steep slopes, leads to dry habitats, shallow soils and seasonality. Humidity regime, related to topography, is stronger than geography in promoting floristic links among limestone vegetation 
communities of the Caribbean where floristic affinities of dry communities were stronger with dry communities on other islands than to the more humid communities on the same island (Trejo-Torres \& Ackerman 2002).

In Brazil, while limestone outcrops within a seasonal climate favours forests (Scariot \& Sevilha 2000, Felfili 2003), quartzites or sandstone rocks (above $900 \mathrm{~m}$ altitude) support predominantly a shrub-herbaceous vegetation called "campos rupestres" or rocky fields, with elevated rainfall and cloudiness (Harley \& Giulietti 2004). In both these vegetation types, species are selected by their drought resistance. Some are restricted to this rocky environment while others are also found in more mesic environments, even in other vegetation types.

The seasonally deciduous forests are distributed in patches in Central Brazil and also in states of Minas Gerais, Bahia and in the Amazonian borders. The seasonally deciduous forests on limestone outcrops show a vegetation that does not differ significantly from the deciduous arboreal "Caatinga" and is rich in succulents from the cactus and bromeliad families (Rizzini 1997).

On limestone outcrops in Minas Gerais, the vegetation is related to the features of the rocks, with an alternating cover of woody vegetation dominated by Anadenanthera colubrina var. cebil (Griseb.) Altschul and Piptadenia gonoacantha (Mart.) Macbr. and scattered dense patches of the bromeliad Encholirium spectabile Mart. (Pedersoli \& Martins 1972). Andrade-Lima (1981) describes the "Caatinga" in Minas Gerais and Bahia as tall forests on limestone outcrops with an association of TabebuiaAspidosperma-Astronium-Cavanillesia.

The seasonally deciduous forests (dry forests) tend to occur in disjunct patches in the neotropics (Pennington et al. 2000) and also in Paranã valley, northeast Goiás State, Brazil (Scariot \& Sevilha 2000) on different soils types including rock outcrops (Felfili 2003). They are composed of trees that are deciduous for longer in the dry season than other tropical forests. The families Bromeliaceae, Compositae, Malvaceae and Marantaceae predominate in the understorey of these forests with the soil only sparsely covered with a few grass species in contrast to savanna vegetation where grasses predominate.

These forests form a tree cover of $50 \%$ to $70 \%$ during the rainy season. The majority of species become leafless in the dry season, when the canopy cover is less than $50 \%$. The trunks are straight with canopy emergents reaching up to 20 to 30 meters. Epiphytes are not very common while Bromeliaceae and Cactaceae are frequent (Felfili 2001).

The objective of this study was to analyze the floristic composition and structure of the tree component in a seasonally deciduous forest on limestone outcrops in Central Brazil and verify floristic affinities with other neo-tropical seasonal forests.

\section{Material and methods}

Study sites - The present work was undertaken in a $c a .50$ hectare patch of seasonally deciduous forest on limestone outcrops at "Fazenda Sabonete", in the municipality of Iaciara, Paranã valley, northeast Goiás State, Brazil $\left(14^{\circ} 03^{\prime} 53.2^{\prime \prime} \mathrm{S}\right.$ and $\left.46^{\circ} 29^{\prime} 15.2^{\prime \prime} \mathrm{W}\right)$. The vegetation of the region is composed of a mosaic of "cerrado" sensu lato, deciduous and semi-deciduous forests (IBGE 1990). The deciduous forests occur on limestone outcrops with steep slopes in patches forming a naturally fragmented vegetation that occurs scattered over Central Brazil and inserted in a matrix of "cerrado" vegetation composed mainly of savannas and grasslands (Felfili 2003).

The forest floor varies from large rock outcrops with trees growing on the rock surface (figure 1) such as Ficus and Commiphora species to trees growing between rocks (figure 2,3) and in the fissures of the rocks (figure 4). Observations on large fallen trees on the forest floor show that the roots are restricted to this shallow layer. Cactus and other succulent plants are also found.

In this region the altitudes vary between $200-500 \mathrm{~m}$, and the slope is gently undulating (3\%-8\%), but with parts steeper and slopes undulating (13\%-20\%) in the hills (Finatec 2001). However some areas have slopes and escarpments steeper than $20 \%$, including the present study site.

Occasionally some stumps were found in the forest, suggesting occasional selective logging of valuable species over time with apparently low impact on the forest structure which appeared mostly undisturbed.

Vegetation sampling - The forest was divided into $20 \mathrm{~m}$ wide transects perpendicular to the longitudinal gradient as suggested by Felfili (1995) for small forested areas with a strong environmental gradient. In this case, the gradient is the slope. The sampling included two phases, firstly four transects were randomly selected, and then $25(20 \times 20 \mathrm{~m})$ plots were randomly distributed within them. The 25 plots $\left(400 \mathrm{~m}^{2}\right)$ were permanently established with iron stakes marking their corners, totaling a sample of 1 ha within the forest (see figure 5).

Girth at breast height $(\mathrm{GBH} \geq 15.7 \mathrm{~cm}$ : diameter at breast height $\mathrm{DBH} \geq 5 \mathrm{~cm}$ ) and total height were measured for all woody individuals which were tagged with aluminium labels. Girth was measured with a metric tape and afterwards was converted into diameter $\left(\mathrm{DBH}=\mathrm{GBH} \mathrm{PI}^{-1}\right)$ and all the following analyses refers to DBH. Height was measured with a telescopic pole graduated in $\mathrm{cm}$. The species were identified and fertile botanical vouchers, collected in the wet and dry seasons, were deposited at IBGE herbarium in Brasilia, DF. Species classification followed Cronquist (1988) but Leguminosae was subdivided into three subfamilies. 

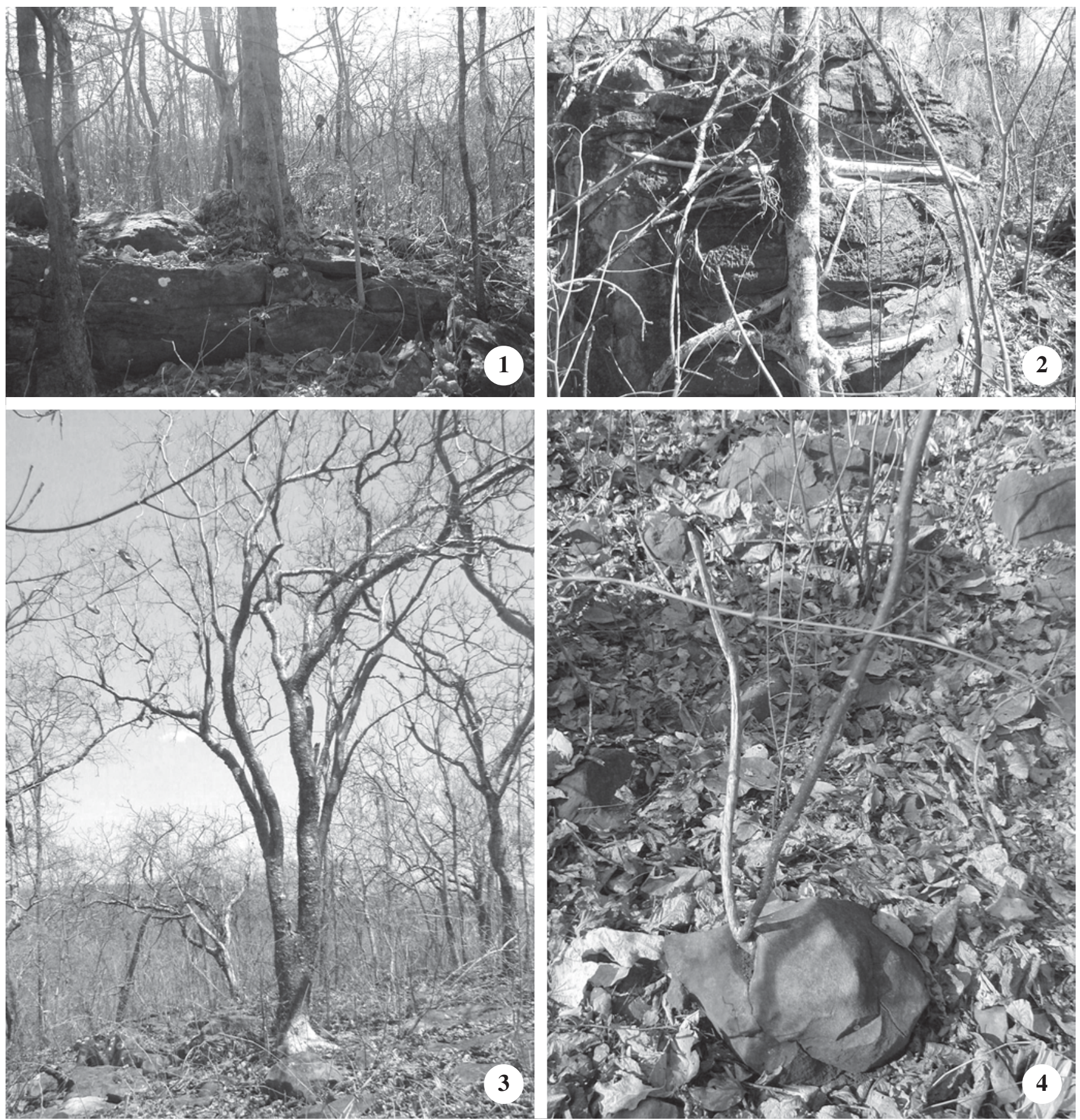

Figure 1. Forest floor covered by a thick layer of dry leaves with trees growing on rocky layer. 2. Ficus growing on large limestone rock outcrops. 3. Commiphora growing on large limestone rock outcrops. 4. Plant growing in a limestone rock fissure. All taken in a seasonally deciduous forest on limestone outcrops at Iaciara, Goiás State, in the Paranã valley, Central Brazil.

Each plot was classified regarding rocky cover according to the following percentage scale: $0 \%-25 \%, 26 \%-50 \%, 51 \%$ $75 \%, 76 \%-100 \%$ rock. Phytosociological parameters (density, frequency, dominance and the importance value index) were calculated (Kent \& Coker 1992).

The species list was compared with published lists for seasonal forests from northeastern ("caatinga"; Giulietti et al. 2002, Mayo 1996), central ("cerrado"; Pereira et al. 1996) and western ("cerrado"; Pott \& Pott 2003) Brazil, and the Chiquitano forest of Bolivia (dry forest; Ibiseli et al. 2002). Synonyms were checked for this comparison. Floristic affinity was related to the occurrence of common species between this fragment in the Paranã Valley and those regional lists, species referred in the literature as endemic were also verified. 


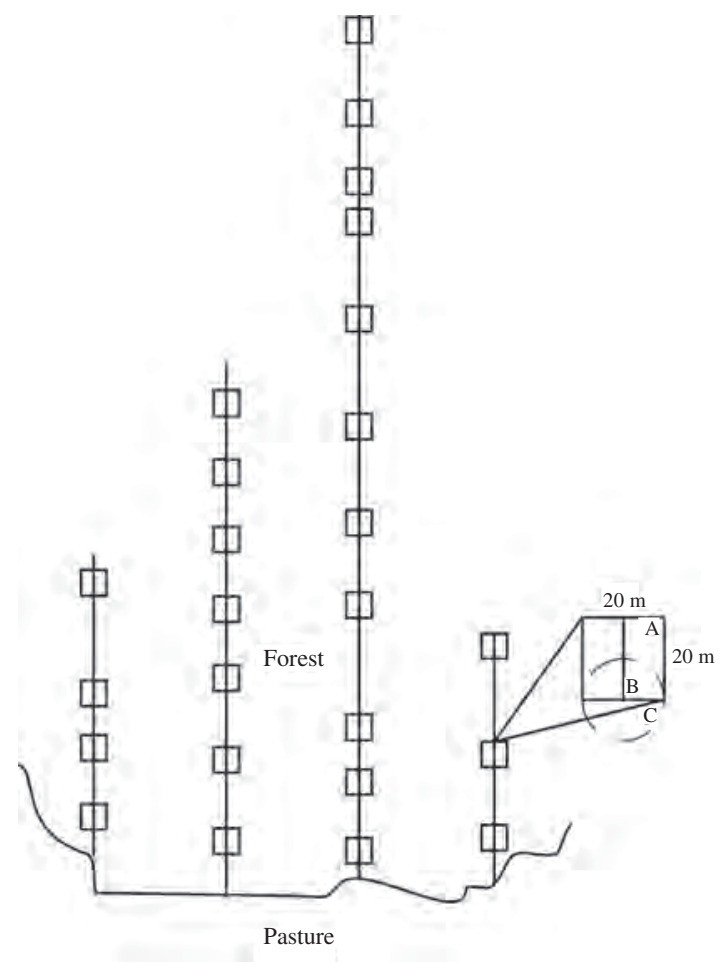

Figure 5. Croquis of the sampling design in a seasonally deciduous forest on limestone outcrops in Iaciara, Goiás State in the Paranã valley, Central Brazil.

\section{Results}

Community floristic composition - A total of 734 individuals with $\mathrm{DBH}>5 \mathrm{~cm}$ were sampled, belonging to 39 tree species, distributed in 19 botanical families (table 1). The Leguminosae family with 11 species was the most represented and characteristic of the area, followed by Bignoniaceae and Apocynaceae with four species each and Bombaceae with three species. These four families had $55 \%$ of all the species. Of the total number of families encountered $(n=19), 30 \%$ are represented by only one species.

From a total of 32 genera, Aspidosperma (four species), Machaerium (four species), Tabebuia (three species) and Bauhinia (two species) represented around $32.5 \%$ of the total number of species in the forest.

Forest structure - In relation to density, only Dilodendron bipinnatum, Acacia tenuifolia (syn. A. paniculata), Pseudobombax tomentosum, Combretum duarteanun and Myracrodruon urundeuva represented 56.4\% of the total number of individuals in the hectare (table 2).

Nine species: Dilodendron bipinnatum, Pseudobombax tomentosum, Myracrodruon urundeuva, Cavanillesia arborea, Acacia tenuifolia, Combretum duarteanum,

Table 1. List of species found per botanical family in a remnant of seasonally deciduous forest on limestone outcrops, in the Iaciara municipality, state of Goiás, Brazil. (ca = "caatinga", car = "carrasco", cer = "cerrado", ce/fe = transition "cerrado"/ forest, $\mathrm{co}=$ "cerradão", end = endemic, $\mathrm{fe}=$ seasonal forest (deciduous and semi-deciduous), $\mathrm{fm}=$ mesophytic forest, ma = mata atlantica, $\mathrm{mc}=$ riparian forest.)

\begin{tabular}{|c|c|c|}
\hline $\begin{array}{l}\text { Family } \\
\text { Species }\end{array}$ & Reference & Type of vegetation \\
\hline \multicolumn{3}{|l|}{ ANACARDIACEAE } \\
\hline \multirow[t]{4}{*}{ Astronium fraxinifolium Schott ex Spreng. } & Mayo (1996) & ma, ca \\
\hline & Pott \& Pott (2003) & fe, co \\
\hline & Ibiseli et al. (2002) & fe \\
\hline & Pereira et al. (1996) & fe \\
\hline \multirow[t]{4}{*}{ Myracrodruon urundeuva Fr. Allem. } & Мayo (1996) & ma, ca \\
\hline & Pott \& Pott (2003) & fe, co \\
\hline & Ibiseli et al. (2002) & fe \\
\hline & Pereira et al. (1996) & $\mathrm{fm}$ \\
\hline \multicolumn{3}{|l|}{$\begin{array}{l}\text { ANNONACEAE } \\
\text { Oxandra reticulata Maas }\end{array}$} \\
\hline \multicolumn{3}{|l|}{ APOCYNACEAE } \\
\hline \multirow{2}{*}{ Aspidosperma parvifolium A. DC. } & Mayo (1996) & ma \\
\hline & Pott \& Pott (2003) & fe \\
\hline \multirow[t]{5}{*}{ Aspidosperma pyrifolium Mart. } & Mayo (1996) & $\mathrm{ca}$ \\
\hline & Giulietti et al. (2002) & end \\
\hline & Pott \& Pott (2003) & fe \\
\hline & Ibiseli et al. (2002) & fe \\
\hline & Pereira et al. (1996) & fe \\
\hline
\end{tabular}


continuation

\begin{tabular}{|c|c|c|}
\hline $\begin{array}{l}\text { Family } \\
\text { Species }\end{array}$ & Reference & Type of vegetation \\
\hline Aspidosperma subincanum Mart. & $\begin{array}{l}\text { Mayo (1996) } \\
\text { Pott \& Pott }(2003) \\
\text { Ibiseli et al. }(2002) \\
\text { Pereira } \text { et al. }(1996)\end{array}$ & $\begin{array}{l}\text { ma } \\
\text { fe } \\
\text { fe } \\
\mathrm{fe}\end{array}$ \\
\hline $\begin{array}{l}\text { BIGNONIACEAE } \\
\text { Jacaranda brasiliana (Lam.) Pers. }\end{array}$ & $\begin{array}{l}\text { Mayo (1996) } \\
\text { Pereira et al. (1996) }\end{array}$ & $\begin{array}{l}\mathrm{ca} \\
\mathrm{fm}, \mathrm{mc}\end{array}$ \\
\hline Tabebuia impetiginosa (Mart. ex DC.) Standl. & $\begin{array}{l}\text { Mayo (1996) } \\
\text { Pott \& Pott }(2003) \\
\text { Ibiseli et al. (2002) }\end{array}$ & $\begin{array}{l}\text { ma } \\
\text { fe, co } \\
\text { fe }\end{array}$ \\
\hline Tabebuia roseo-alba (Ridley) Sandw. & $\begin{array}{l}\text { Mayo (1996) } \\
\text { Pott \& Pott (2003) } \\
\text { Ibiseli et al. (2002) }\end{array}$ & $\begin{array}{l}\text { fe, co } \\
\text { fe }\end{array}$ \\
\hline Tabebuia serratifolia (Vahl) Nicholson & $\begin{array}{l}\text { Mayo (1996) } \\
\text { Ibiseli et al. }(2002) \\
\text { Pereira et al. (1996) }\end{array}$ & $\begin{array}{l}\mathrm{ma} \\
\mathrm{fe} \\
\mathrm{fe}\end{array}$ \\
\hline $\begin{array}{l}\text { BOMBACACEAE } \\
\text { Cavanillesia arborea (Willd.) K. Schum. } \\
\text { Pseudobombax tomentosum (Mart. \& Zucc.) A. Robyns } \\
\text { Chorisia speciosa A. St.-Hil. }\end{array}$ & $\begin{array}{l}\text { Mayo (1996) } \\
\text { Pereira et al. (1996) }\end{array}$ & $\begin{array}{l}\text { ca, ma } \\
\text { ce / fe }\end{array}$ \\
\hline $\begin{array}{l}\text { BURSERACEAE } \\
\text { Commiphora leptophloeos (Mart.) J.B. Gillett }\end{array}$ & $\begin{array}{l}\text { Giulietti et al. (2002) } \\
\text { Pott \& Pott (2003) } \\
\text { Ibiseli et al. }(2002)\end{array}$ & $\begin{array}{l}\text { end } \\
\text { fe } \\
\text { fe }\end{array}$ \\
\hline $\begin{array}{l}\text { COMBRETACEAE } \\
\text { Combretum duarteanum Cambess. }\end{array}$ & Mayo (1996) & car \\
\hline $\begin{array}{l}\text { ERYTHROXYLACEAE } \\
\text { Erythroxylum daphnites Mart. }\end{array}$ & $\begin{array}{l}\text { Ibiseli et al. (2002) } \\
\text { Pereira et al. (1996) }\end{array}$ & $\begin{array}{l}\mathrm{fe} \\
\mathrm{ce} / \mathrm{fe}\end{array}$ \\
\hline $\begin{array}{l}\text { FLACOURTIACEAE } \\
\text { Casearia rupestris Eichler }\end{array}$ & $\begin{array}{l}\text { Pott \& Pott }(2003) \\
\text { Pereira et al. }(1996)\end{array}$ & $\begin{array}{l}\text { fe, co } \\
\text { fe }\end{array}$ \\
\hline $\begin{array}{l}\text { LEGUMINOSAE CAESALPINOIDEAE } \\
\text { Bauhinia membranacea Benth. } \\
\text { Bauhinia ungulata } \text { L. }\end{array}$ & $\begin{array}{l}\text { Mayo (1996) } \\
\text { Mayo (1996) } \\
\text { Ibiseli et al. (2002) }\end{array}$ & $\begin{array}{l}\mathrm{ma} \\
\mathrm{ma} \\
\mathrm{fe}\end{array}$ \\
\hline $\begin{array}{l}\text { LEGUMINOSAE MIMOSOIDEAE } \\
\text { Acacia tenuifolia }(\mathrm{L} .) \text { Willd. }\end{array}$ & $\begin{array}{l}\text { Mayo (1996) } \\
\text { Giulietti et al. (2002) } \\
\text { Pott \& Pott (2003) } \\
\text { Ibiseli et al. }(2002)\end{array}$ & $\begin{array}{l}\text { ma } \\
\text { ca } \\
\text { fe, co } \\
\text { fe }\end{array}$ \\
\hline Anadenanthera colubrina var. cebil (Griseb.) Altschul & $\begin{array}{l}\text { Mayo (1996) } \\
\text { Pott \& Pott (2003) } \\
\text { Ibiseli et al. }(2002)\end{array}$ & $\begin{array}{l}\text { ca, cer } \\
\text { fe } \\
\text { fe }\end{array}$ \\
\hline Piptadenia gonoacantha (Mart.) Macbr. & $\begin{array}{l}\text { Mayo (1996) } \\
\text { Pott \& Pott (2003) }\end{array}$ & $\begin{array}{l}\mathrm{ca} \\
\mathrm{fe}\end{array}$ \\
\hline $\begin{array}{l}\text { LEGUMINOSAE PAPILIONOIDEAE } \\
\text { Erythrina verna Vell. }\end{array}$ & $\begin{array}{l}\text { Mayo (1996) } \\
\text { Pereira et al. (1996) }\end{array}$ & $\begin{array}{l}\text { ca } \\
\text { fe }\end{array}$ \\
\hline
\end{tabular}


continuation

\begin{tabular}{|c|c|c|}
\hline $\begin{array}{l}\text { Family } \\
\text { Species }\end{array}$ & Reference & Type of vegetation \\
\hline Machaerium aculeatum Raddi & $\begin{array}{l}\text { Mayo (1996) } \\
\text { Pott \& Pott }(2003) \\
\text { Pereira et al. }(1996)\end{array}$ & $\begin{array}{l}\mathrm{ma} \\
\mathrm{fe}, \mathrm{fm} \\
\mathrm{fe}\end{array}$ \\
\hline Machaerium acutifolium Vogel & $\begin{array}{l}\text { Mayo (1996) } \\
\text { Pott \& Pott (2003) } \\
\text { Ibiseli et al. (2002) }\end{array}$ & $\begin{array}{l}\text { ca, cer } \\
\text { co } \\
\text { co }\end{array}$ \\
\hline $\begin{array}{l}\text { Machaerium stipitatum (DC.) Vogel } \\
\text { Machaerium scleroxylon Tul. }\end{array}$ & $\begin{array}{l}\text { Mayo (1996) } \\
\text { Pott \& Pott (2003) } \\
\text { Ibiseli et al. (2002) }\end{array}$ & $\begin{array}{l}\mathrm{ma} \\
\mathrm{fm} \\
\mathrm{fe}\end{array}$ \\
\hline $\begin{array}{l}\text { Machaerium cf. oblongifolium Vogel } \\
\text { Lonchocarpus montanus Tozzi }\end{array}$ & Mayo (1996) & $\mathrm{ma}$ \\
\hline $\begin{array}{l}\text { MALPIGHIACEAE } \\
\text { Indet. }\end{array}$ & & \\
\hline $\begin{array}{l}\text { OLACACEAE } \\
\text { Ximenia americana } \mathrm{L} \text {. }\end{array}$ & $\begin{array}{l}\text { Mayo (1996) } \\
\text { Ibiseli et al. (2002) }\end{array}$ & $\begin{array}{l}\mathrm{ca} \\
\mathrm{fe}\end{array}$ \\
\hline $\begin{array}{l}\text { RHAMNACEAE } \\
\text { Rhamnidium elaeocarpum Reiss. }\end{array}$ & $\begin{array}{l}\text { Mayo (1996) } \\
\text { Pott \& Pott }(2003) \\
\text { Ibiseli et al. }(2002) \\
\text { Pereira et al. }(1996)\end{array}$ & $\begin{array}{l}\mathrm{ca} \\
\mathrm{fe} \\
\mathrm{fe} \\
\mathrm{fe}\end{array}$ \\
\hline $\begin{array}{l}\text { RUBIACEAE } \\
\text { Guettarda viburnoides Cham. \& Schltdl. }\end{array}$ & $\begin{array}{l}\text { Mayo (1996) } \\
\text { Pott \& Pott }(2003) \\
\text { Ibiseli et al. }(2002)\end{array}$ & $\begin{array}{l}\text { ma } \\
\text { co } \\
\text { co }\end{array}$ \\
\hline Tocoyena formosa (Cham. \& Schltdl.) K. Schum. & $\begin{array}{l}\text { Mayo (1996) } \\
\text { Ibiseli et al. (2002) }\end{array}$ & $\begin{array}{l}\text { cer, car } \\
\text { co }\end{array}$ \\
\hline $\begin{array}{l}\text { SAPINDACEAE } \\
\text { Dilodendron bipinnatum Radlk. }\end{array}$ & $\begin{array}{l}\text { Pott \& Pott (2003) } \\
\text { Ibiseli et al. (2002) }\end{array}$ & $\begin{array}{l}\text { fe, co } \\
\text { co }\end{array}$ \\
\hline $\begin{array}{l}\text { SAPOTACEAE } \\
\text { Micropholis venulosa (Mart. \& Eichl.) Pierre }\end{array}$ & & \\
\hline $\begin{array}{l}\text { STERCULIACEAE } \\
\text { Guazuma ulmifolia Lam. }\end{array}$ & $\begin{array}{l}\text { Mayo (1996) } \\
\text { Pott \& Pott }(2003) \\
\text { Ibiseli et al. }(2002) \\
\text { Pereira } \text { et al. }(1996)\end{array}$ & $\begin{array}{l}\text { ma } \\
\text { fe, co, mc } \\
\text { fe } \\
\text { fe }\end{array}$ \\
\hline Sterculia striata A. St.-Hil \& Naud. & $\begin{array}{l}\text { Mayo (1996) } \\
\text { Pott \& Pott }(2003) \\
\text { Ibiseli et al. }(2002) \\
\text { Pereira } \text { et al. }(1996)\end{array}$ & $\begin{array}{l}\text { ma } \\
\text { fe, co } \\
\text { co } \\
\text { fm }\end{array}$ \\
\hline $\begin{array}{l}\text { TILIACEAE } \\
\text { Luehea candicans Mart. \& Zucc. }\end{array}$ & $\begin{array}{l}\text { Mayo (1996) } \\
\text { Ibiseli et al. (2002) }\end{array}$ & $\begin{array}{l}\text { ma } \\
\text { cer }\end{array}$ \\
\hline $\begin{array}{l}\text { VOCHYSIACEAE } \\
\text { Callisthene fasciculata Mart. }\end{array}$ & $\begin{array}{l}\text { Mayo (1996) } \\
\text { Pott \& Pott (2003) } \\
\text { Ibiseli et al. }(2002) \\
\text { Pereira } \text { et al. (1996) }\end{array}$ & $\begin{array}{l}\text { car } \\
\text { fe, co } \\
\text { co } \\
\text { fm }\end{array}$ \\
\hline
\end{tabular}


Table 2. Density (Absolute and Relative) values, Frequency (Absolute and Relative), Dominance (Absolute and Relative) and Importance value index of trees with DAP $>5 \mathrm{~cm}$ in a seasonally deciduous forest on limestone outcrops, Municipality of Iaciara, state of Goiás, Brazil.

\begin{tabular}{|c|c|c|c|c|c|c|c|}
\hline Species & $\begin{array}{l}\text { Abs. } \\
\text { Dens. }\end{array}$ & $\begin{array}{c}\text { Rel. } \\
\text { Dens. }\end{array}$ & $\begin{array}{l}\text { Abs. } \\
\text { Dom. }\end{array}$ & $\begin{array}{l}\text { Rel. } \\
\text { Dom. }\end{array}$ & $\begin{array}{c}\text { Abs. } \\
\text { Fr. }\end{array}$ & $\begin{array}{l}\text { Rel. } \\
\text { Fr. }\end{array}$ & IVI \\
\hline Dilodendron bipinnatum & 113.54 & 15.46 & 1.84 & 11.24 & 88 & 7.84 & 34.53 \\
\hline Pseudobombax tometosum & 76.04 & 10.35 & 2.35 & 14.35 & 79 & 7.09 & 31.79 \\
\hline Myracrodruon urundeuva & 61.46 & 8.37 & 1.26 & 7.71 & 88 & 7.84 & 23.92 \\
\hline Cavanillesia arborea & 6.25 & 0.85 & 3.26 & 19.95 & 21 & 1.87 & 22.67 \\
\hline Acacia tenuifolia & 92.71 & 12.62 & 0.51 & 3.17 & 71 & 6.34 & 22.14 \\
\hline Combretum duarteanum & 70.83 & 9.65 & 0.40 & 2.49 & 71 & 6.34 & 18.48 \\
\hline Tabebuia impetiginosa & 35.42 & 4.82 & 1.36 & 8.32 & 58 & 5.22 & 18.36 \\
\hline Sterculia striata & 31.25 & 4.26 & 1.21 & 7.39 & 75 & 6.72 & 18.36 \\
\hline Dead trees & 40.63 & 5.53 & 0.45 & 2.76 & 63 & 5.60 & 13.89 \\
\hline Aspidosperma subincanum & 27.08 & 3.69 & 0.60 & 3.70 & 58 & 5.22 & 12.61 \\
\hline Astronium fraxinifolium & 17.71 & 2.41 & 0.61 & 3.74 & 42 & 3.73 & 9.88 \\
\hline Guazuma ulmifolia & 29.17 & 3.97 & 0.17 & 1.09 & 42 & 3.73 & 8.79 \\
\hline Commiphora leptophloeos & 11.46 & 1.56 & 0.52 & 3.21 & 29 & 2.61 & 7.38 \\
\hline Lonchocarpus montanus & 12.50 & 1.70 & 0.32 & 1.99 & 29 & 2.61 & 6.30 \\
\hline Tabebuia roseo-alba & 11.46 & 1.56 & 0.11 & 0.72 & 38 & 3.36 & 5.64 \\
\hline Piptadenia gonoacantha & 12.50 & 1.70 & 0.07 & 0.43 & 33 & 2.99 & 5.12 \\
\hline Aspidosperma pyrifolium & 9.38 & 1.28 & 0.12 & 0.78 & 33 & 2.99 & 5.04 \\
\hline Anadenanthera colubrina & 6.25 & 0.85 & 0.14 & 0.91 & 21 & 1.87 & 3.63 \\
\hline Machaerium stipitatum & 7.29 & 0.99 & 0.13 & 0.80 & 17 & 1.49 & 3.29 \\
\hline Malpighiaceae & 8.33 & 1.13 & 0.05 & 0.34 & 17 & 1.49 & 2.97 \\
\hline Machaerium oblongifolium & 5.21 & 0.71 & 0.04 & 0.30 & 17 & 1.49 & 2.50 \\
\hline Luehea candicans & 10.42 & 1.42 & 0.08 & 0.49 & 4 & 0.37 & 2.28 \\
\hline Aspidosperma parvifolium & 5.21 & 0.71 & 0.11 & 0.70 & 8 & 0.75 & 2.16 \\
\hline Jacaranda brasiliana & 4.17 & 0.57 & 0.02 & 0.14 & 13 & 1.12 & 1.83 \\
\hline Machaerium scleroxylon & 2.08 & 0.28 & 0.12 & 0.77 & 8 & 0.75 & 1.80 \\
\hline Erythroxylum daphnites & 3.13 & 0.43 & 0.01 & 0.08 & 13 & 1.12 & 1.63 \\
\hline Chorisia speciosa & 1.04 & 0.14 & 0.15 & 0.96 & 4 & 0.37 & 1.48 \\
\hline Bauhinia ungulata & 3.13 & 0.43 & 0.01 & 0.09 & 8 & 0.75 & 1.26 \\
\hline Ximenia americana & 2.08 & 0.28 & 0.03 & 0.22 & 8 & 0.75 & 1.25 \\
\hline Machaerium acutifolium & 2.08 & 0.28 & 0.02 & 0.13 & 8 & 0.75 & 1.16 \\
\hline Tocoyena formosa & 2.08 & 0.28 & 0.01 & 0.07 & 8 & 0.75 & 1.10 \\
\hline Rhamnidium elaeocarpum & 2.08 & 0.28 & 0.00 & 0.05 & 8 & 0.75 & 1.08 \\
\hline Machaerium aculeatum & 1.04 & 0.14 & 0.06 & 0.38 & 4 & 0.37 & 0.90 \\
\hline Guettarda viburnoides & 2.08 & 0.28 & 0.00 & 0.05 & 4 & 0.37 & 0.71 \\
\hline Micropholis venulosa & 1.04 & 0.14 & 0.02 & 0.13 & 4 & 0.37 & 0.64 \\
\hline Tabebuia serratifolia & 1.04 & 0.14 & 0.01 & 0.12 & 4 & 0.37 & 0.64 \\
\hline Erythrina verna & 1.04 & 0.14 & 0.01 & 0.07 & 4 & 0.37 & 0.58 \\
\hline Oxandra reticulata & 1.04 & 0.14 & 0.01 & 0.07 & 4 & 0.37 & 0.58 \\
\hline Callisthene fasciculata & 1.04 & 0.14 & 0.00 & 0.03 & 4 & 0.37 & 0.55 \\
\hline Casearia rupestris & 1.04 & 0.14 & 0.00 & 0.02 & 4 & 0.37 & 0.53 \\
\hline Bauhinia membranaceae & 1.04 & 0.14 & 0.00 & 0.02 & 4 & 0.37 & 0.53 \\
\hline Total & 734.38 & 100 & 16.73 & 100 & 1.117 & 100 & 300 \\
\hline
\end{tabular}


Tabebuia impetiginosa, Sterculia striata and Aspidosperma subincanum had the greatest importance values in the forest, representing around $72.25 \%$ of the total.

The basal area of the forest was $16.37 \mathrm{~m}^{2}$ per hectare, with species Cavanillesia arborea $\left(3.26 \mathrm{~m}^{2} \mathrm{ha}^{-1}\right)$, Pseudobombax tomentosum $\left(2.35 \mathrm{~m}^{2} \mathrm{ha}^{-1}\right)$, Dilodendron bipinnatum $\left(1.84 \mathrm{~m}^{2} \mathrm{ha}^{-1}\right)$, Tabebuia impetiginosa $(1.36$ $\left.\mathrm{m}^{2} \mathrm{ha}^{-1}\right)$ and Myracrodruon urundeuva $\left(1.26 \mathrm{~m}^{2} \mathrm{ha}^{-1}\right)$ the most important, occupying together $61.5 \%$ of the total basal area (table 2). Of these, only Dilodendron bipinnatum had individual trees of smaller sizes, and was in the most abundant group because of its high density found in the hectare (113 individuals ha ${ }^{-1}$ ). The dead trees still standing represented $5.48 \%$ of the relative density.

Of the largest trees, individuals of Cavanillesia arborea attained $120 \mathrm{~cm} \mathrm{DBH}$ while more than $95 \%$ were under $50 \mathrm{~cm}$. The height of the species varied from 3.5 $\mathrm{m}$ for individuals in the understorey to around $15 \mathrm{~m}$ for the canopy species and for emerging individuals such as Astronium fraxinifolium and Cavanillesia arborea, which were the largest trees sampled. The majority of the individuals had heights around $13 \mathrm{~m}$, and only species of great stature and emerging canopy individuals were above this height.

Rocky cover was above $75 \%$ for most plots and in all plots many trees of all species grew on rocks or in fissures showing their capacity to survive in a stressful environment.

\section{Discussion}

The trees in our study site grow on a shallow substrate, endure a long dry season (5 to 7 months without rain) and develop strategies to cope with those conditions such as shedding their leaves during the period - some relying on water storage in the roots or trunks such as Cavanillesia arborea, Commiphora leptophloeos - while many herbs and shrubs are ephemeral. High cover of ephemerals is common at the understorey of other seasonal forests worldwide such as the monsoon seasonal forests on limestone outcrops in China (Cao \& Zhang 1997). The rocks become covered in a thick layer of dry leaves that will be the organic matter for the next growing season (figure 1A). The richness of the calcareous rocks associated with abundant organic matter most likely supports the forest growth every year.

The floristic composition and structure resembles the ecological unit suggested by Andrade-Lima (1981) for the "caatinga". He described it as a high forest of "caatinga" occurring on limestone outcrops in the north of Minas Gerais and Bahia States having Cavanillesia arborea as a typical species. He classified this type as the Tabebuia-Aspidosperma-Astronium-Cavanillesia association. All those genera are present in the Iaciara forest, reinforcing the hypothesis that this remnant in Central Brazil has strong affinities with the larger seasonal formations of South America. Some species found in these forests also occurred in "cerrado", "carrasco" or Atlantic forests in other places, (see table 1).

Several widespread species were common with the flora from the "caatinga" and associated forests from Northeastern Brazil (Giulietti et al. 2002, Mayo 1996), including two species classified by Giulietti et al. (2002) as endemic of the "caatinga", Aspidosperma pyrifolium and Commiphora leptophloeos (see table 1). This forest also shared several species with the list of Pott \& Pott (2003) from Mato Grosso do Sul State including the Pantanal, and to Chiquitano forest of Bolivia (Ibiseli et al. 2002), to APA de Cafuringa, seasonally dry forest in the Federal District by Pereira et al. (1996) (see table 1), supporting the suggestion of a strong floristic link between the fragments in Central Brazil and other seasonally dry forests in the neotropics. These support the suggestion of Pennington et al. (2000) that there is strong affinity between the seasonal forests of South America, and also that of Felfili (2003) that the patches of seasonal forests within Central Brazil act as stepping stones which approximate the larger seasonal formations of the "caatinga" with the Chaco. The latter cites patches of seasonal forests scattered throughout Central Brazil, based on data from IBGE vegetation map (www.ibge.org.br accessed in 29/07/2006) and explains that these areas - classified by IBGE as Seasonal Forests or Seasonal Forest/Savanna contact zones - either contain only seasonal forests or patches of seasonal forests inserted into a savanna matrix. As the fragments are scattered through the central region, going east-west, they would function as stepping stones for the species from the larger extensions of seasonally dry deciduous forests on both sides. Many species are wind dispersed in those forests making possible the gene flow between natural fragments surrounded by savanna vegetation. Animals forage in different vegetation types within a region thus facilitating seed dispersion and pollination among the fragments.

In this forest, the legume family was dominant, which is commonly found in the neo-tropical forests including the seasonal forests (Gentry 1995, Pennington et al. 2000). The estimates of species richness and their density per hectare are within the values found for seasonal forests elsewhere (table 3 ).

The maximum sizes found in these forests, especially their heights, place the arboreal species of this forest on 
Table 3. Estimates of richness, density and basal area per hectare of seasonal forests from various localities (Dens. (ha) absolute density per hectare, $\mathrm{G}\left(\mathrm{m}^{2} \mathrm{ha}^{-1}\right)$ - basal area in $\left.\mathrm{m}^{2} \mathrm{ha}^{-1}\right)$

\begin{tabular}{|c|c|c|c|c|c|c|}
\hline Forest type & $\phi \underset{(\mathrm{cm})}{\operatorname{minimum}}$ & $\begin{array}{c}\text { № of } \\
\text { species }\end{array}$ & $\begin{array}{l}\text { Dens. } \\
\text { (ha) }\end{array}$ & $\begin{array}{c}\mathrm{G} \\
\left(\mathrm{m}^{2} \mathrm{ha}^{-1}\right)\end{array}$ & Locality & Source \\
\hline Seasonally deciduous & 5 & 39 & 734.0 & 16.73 & Iaciara, GO, Brazil & This study \\
\hline Seasonally deciduous & 5 & 56 & 633.0 & 19.36 & $\begin{array}{l}\text { Monte Alegre, GO, } \\
\text { Brazil }\end{array}$ & $\begin{array}{l}\text { Nascimento et al. } \\
\text { (2004) }\end{array}$ \\
\hline Semi deciduous & 5 & 72 & 510.0 & 20.0 & $\begin{array}{l}\text { Nova Xavantina, } \\
\text { MT, Brazil }\end{array}$ & Felfili et al. (1998) \\
\hline Seasonally deciduous & 10 & 55 & 333.0 & 28.7 & Barinas, Venezuela & Lamprecht (1990) \\
\hline Seasonally deciduous & 5 & 44 & 591.0 & 23.17 & $\begin{array}{l}\text { São Domingos, GO, } \\
\text { Brazil }\end{array}$ & $\begin{array}{l}\text { Scariot \& Sevilha } \\
(2000)\end{array}$ \\
\hline Seasonally deciduous & 3.2 & 45 & 1924.0 & 15.86 & Macaiba, RN, Brazil & $\begin{array}{l}\text { Cestaro \& Soares } \\
(2004)\end{array}$ \\
\hline Seasonally deciduous & 3.2 & 56 & 1587.0 & 15.88 & Macaiba, RN, Brazil & $\begin{array}{l}\text { Cestaro \& Soares } \\
(2004)\end{array}$ \\
\hline Semi deciduous & 5 & 101 & 842.2 & 12.53 & Piracicaba, SP, Brazil & $\begin{array}{l}\text { Viana \& Tabanez } \\
\text { (1996) }\end{array}$ \\
\hline
\end{tabular}

limestone outcrops as Mesophanerophytes according to Raunkier's classification (Kent \& Coker 1992). The basal area of $16.37 \mathrm{~m}^{2} \mathrm{ha}^{-1}$ places it within the range of "cerradão" basal area (Felfili et al. 1994), lower than most gallery and Amazonian forests that usually show basal area between 20 and $40 \mathrm{~m}^{2}$ ha $^{-1}$ (Felfili 1995). Although slightly less, the values of basal area per hectare can be considered within that found for this type of vegetation (see table 3 ).

The percentage of dead standing trees can be considered within the range of undisturbed seasonal forests (Oliveira Filho et al. 1997, Araújo 2002) and even lower than the figures found for some "cerrado" sites (Felfili et al. 1994, 1997, 2001). The large sizes of some trees, the low rate of dead standing trees, and the presence of a large number of small individuals, suggest that an occasional tree felling over time did not cause a great disturbance in this forest.

However, these patches of seasonal forests on limestone outcrops present high densities of timber species such as Myracrodruon urundeuva (61.0 ind. ha- ${ }^{-1}$ ), Tabebuia impetiginosa (35.0 ind. ha ${ }^{-1}$ ) and Aspidosperma subincanum (27.0 ind. ha ${ }^{-1}$ ) and need conservation and management that consider the singular aspects of these communities. The selective extraction of timber species elsewhere, and the absence of sustainable management practices is selectively eroding the natural reserves of these species and can compromise the existence and conservation of these populations in these scattered communities in the "cerrado" biome. Mining of calcareous rocks destroys entire forests. These can have impact on the genetic flux throughout the seasonal forests of South America, proposed by Pennington et al. (2000) as a new phytogeographic unit.

This study showed that the vegetation on limestone outcrops at the Paranã valley in Goiás is a mesophanerophyte deciduous forest, composed of valuable timber species and sharing several common species with the neotropical seasonal forests, especially those of Pantanal, Chiquitania forests and "caatinga". Further studies at regional level, including the sampling of the various patches of this vegetation scattered through the limestone outcrops of Central Brazil should be conducted to verify the floristic similarities among the seasonal forests of the neotropics and the specific traits of these forests on limestone outcrops.

Acknowledgements - This work is part of the project "Estratégias para a Conservação e Manejo da Biodiversidade em Fragmentos de Florestas Semidecíduas" financed by Probio and CNPq which was supported in the follow up grants from DFID-UK. We thank CNPq/Pibic and Capes for the grants awarded; Sr. Silvio Lacerda, owner of "Fazenda Sabonete" in Iaciara, Goiás State, for permission to work on his farm; Roberta C. Mendonça, Benedito A. Pereira and Marina Fonseca for help in identifying the botanical material collected; Edson Cardoso, Newton Rodrigues and José Deassis Alvarenga for help in data collection; Julio Sam for editing the figures and to two anonymous referees for their valuable suggestions. 


\section{References}

ALDER, D. 1995. Growth modelling for mixed tropical forests. Tropical Forestry Papers, Oxford Forestry Institute, Oxford.

ANDRADE-LIMA, D. 1981. The caatinga dominium. Revista Brasileira de Botânica 4:149-153.

ARAÚJO, M.M. 2002. Vegetação e mecanismos de regeneração em fragmento de Floresta Estacional Decidual Ripária, Cachoeira do Sul, RS, Brasil. Tese de doutorado, Universidade Federal de Santa Maria, Santa Maria.

BRAUN-BLANQUET, J. 1979. Fitosociologia: base para el estudio de las comunidades vegetales. H. Blume Ediciones, Madrid.

CAO, M. \& ZHANG, J. 1997. Tree species diversity of tropical forest vegetation in Xishuangbanna, SW China. Biodiversity and Conservation 6:995-1006.

CESTARO, L.A. \& SOARES, J.J. 2004. Variações florísticas estrutural e relações fitogeográficas de um fragmento de floresta decídua no Rio Grande do Norte, Brasil. Acta Botanica Brasilica 18:203-218.

CRONQUIST, A. 1988. The evolution and classification of flowering plants. $2^{\text {nd }}$ ed. The New York Botanical Garden, New York.

CROWTHER, J. 1987. Ecological observations in Tropical Karst Terrain, West Malaysia. III. Dynamics of the vegetationsoil-bedrock system. Journal of Biogeography 14:157-164.

FELFILI, J.M. 1995. Diversity, structure and dynamics of a gallery forest in Central Brazil. Vegetatio 117:1-15.

FELFILI, J.M. 1997. Diameter and height distribuitions in a gallery forest community and some of its main species in Central Brazil over a six-year period (1985-1991). Revista Brasileira de Botânica 20:155-162.

FELFILI, J.M. 2001. As principais fisionomias do Espigão Mestre do São Francisco. In Biogeografia do bioma cerrado: estudo fitofisionômico da Chapada do Espigão Mestre do São Francisco (J.M. Felfili \& M.C. Silva Junior, orgs.). Universidade de Brasília, Brasília, p.18-30.

FELFILI, J.M. 2003. Fragmentos de florestas estacionais do Brasil Central: diagnóstico e proposta de corredores ecológicos. In Fragmentação florestal e alternativas de desenvolvimento rural na Região Centro-Oeste (R.B. Costa, org.). Universidade Católica Dom Bosco, Campo Grande, p.139-160.

FELFILI, J.M., FILGUEIRAS, T.S., HARIDASSAN, M., SILVA JUNIOR, M.C., MENDONÇA, R. \& REZENDE, A.V. 1994. Projeto biogeografia do bioma cerrado: vegetação e solos. Caderno de Geociências do IBGE 12:75-166.

FELFILI, J.M., SILVA JUNIOR, M.C., REZENDE, A.V., NOGUEIRA, P.E., WALTER, B.M.T., SILVA, M.A. \& ENCINAS, J.I. 1997. Comparação florística e fitossociológica do cerrado nas Chapadas Pratinha e dos Veadeiros. In Contribuição ao conhecimento ecológico do cerrado (L. Leite \& C.H. Saito, eds.) Universidade de Brasília, Brasília, p.6-11.
FELFILI, J.M., SILVA JUNIOR, M.C. \& NOGUEIRA, P.E. 1998. Levantamento da vegetação arbórea na região de Nova Xavantina, MT. Boletim do Herbário Ezechias Paulo Heringer 3:63-81.

FELFILI, J.M., SILVA JUNIOR, M.C., SEVILHA, A.C., REZENDE, A.V., NOGUEIRA, P.E., WALTER, B.M.T., SILVA, F.C. \& SALGADO, M.A. 2001 Fitosociologia da vegetação arbórea. In Biogeografia do bioma cerrado: estudo fitofisionômico da Chapada do Espigão Mestre do São Francisco (J.M. Felfili \& M.C. Silva Junior, orgs.) Universidade de Brasília, Brasília, p.35-61.

FINATEC. 2001. Inventário hidrelétrico da Bacia do Rio Paranã. Mapas de vegetação, solos e geologia. Finatec/ Agência Nacional de Energia Elétrica, Brasília.

GENTRY, A.H. 1995. Diversity and floristic composition of neotropical dry forests. In Seasonally dry tropical forests (S.H. Bullock, H.A. Mooney \& E. Medina eds.). Cambridge University Press, New York, p.146-194.

GIULIETTI, A.M., HARLEY, R.M., QUEIROZ, L.P., BARBOSA, M.R.V., NETA, A.L.B. \& FIGUEIREDO, M.A. 2002. Espécies endêmicas da Caatinga. In Vegetação e flora da Caatinga (E.V.S.B. Sampaio, A.M. Giulietti, J. Virgínio \& C.F.L. Gamarra-Rojas, eds.) Associação Plantas do Nordeste, Recife, p.103-105

HARLEY, R.M. \& A.M. GIULIETTI. 2004. Wild flowers of the Chapada Diamantina. Rima, São Carlos.

IBGE. 1990. Mapa da vegetação brasileira. Instituto Brasileiro de Geografia e Estatística, Rio de Janeiro.

IBISELI, P.L., COLUMBA, K. \& REICHLE, S. (eds.) 2002. Plan de conservacion y dessarollo sostenible para el Bosque Seco Chiquitano, Cerrado y Pantanal Boliviano. Editora FAN, Santa Cruz de la Sierra.

KENT, M. \& COKER, P. 1992. Vegetation description and analysis - a practical approach. Belhaven Press, London.

LAMPRECHT, H. 1990. Silvicultura nos trópicos: ecossistemas florestais e respectivas espécies arbóreas - possibilidades e métodos de aproveitamento sustentado. GTZ, Eschborn.

MAYO, S.J. 1996. Fanerógamas. In Pesquisa botânica nordestina: progresso e perspectivas. (E.V.S.B. Sampaio, S.J. Mayo \& M.R. Barbosa, eds.). Sociedade Botânica do Brasil, Recife, p.123-144.

NASCIMENTO, A.R.T., FELFILI, J.M. \& MEIRELLES, E.M.L. 2004. Florística e estrutura de um remanescente de Floresta Estacional Decidual de encosta no município de Monte Alegre, GO, Brasil. Acta Botanica Brasilica 18:659-669.

OLIVEIRA FILHO, A.T., MELLO, J.M. \& SCOLFORO, J. R.S. 1997. Effects of past disturbance and edges on tree community structure and dynamics within a fragment of tropical semideciduous forest in south-eastern Brazil over a five-year period (1987-1992). Plant Ecology 131:45-66.

OOSTING, H.J. 1956. The study of plant communities. W.H. Freeman, San Francisco.

PEDERSOLI, J.L. \& MARTINS, J.L. 1972. A vegetação dos afloramentos de calcário. Oreades 5:27-29. 
PENNINGTON, R.T., PRADO, D.E. \& PENDRY, C.A. 2000. Neotropical seasonally dry forests and quaternary vegetation changes. Journal of Biogeography 27:261273.

PEREIRA, B.A.S., MECENAS, V.V., LEITE, F.Q. \& CARDOSO, E.S. 1996. APA da Cafuringa: o retrato do cerrado. Paralelo 15 Editores, Brasília.

PÉREZ-GARCÍA, E. \& MEAVE, J. 2005. Heterogeneity of xerophytic vegetation of limestone outcrops in a tropical deciduous forest region in southern Mexico. Plant Ecology 175:147-163.

PÉREZ-GARCÍA, E.A. \& MEAVE, J.A. 2006. Coexistence and divergence of tropical dry forests and savannas in southern Mexico. Journal of Biogeography 33:438-447.

POTT, A. \& POTT, V.J. 2003. Espécies de fragmentos florestais do Mato Grosso do Sul. In Fragmentação florestal e alternativas de desenvolvimento rural na Região Centro-Oeste (R.B. Costa, org.). Universidade Católica Dom Bosco, Campo Grande, p.27-52.

PRADO, D.E. \& GIBBS, P.E. 1993. Patterns of species distributions in the dry seasonal forests of South America. Annals of the Missouri Botanical Garden 80:902-927.
RIZZINI, C.T. 1997. Tratado de fitogeografia do Brasil aspectos ecológicos, sociológicos e florísticos. $2^{\underline{a}}$ ed. Âmbito Cultural Edições, Rio de Janeiro.

RODAL, M.J.N., SAMPAIO, E.V.S.B. \& FIGUEIREDO, M.A. 1992. Manual sobre métodos de estudo florístico e fitossociológico - Ecossistema Caatinga. Sociedade Botânica do Brasil/CNPq, Brasília.

SCARIOT, A. \& SEVILHA, A.C. 2000. Diversidade, estrutura e manejo de florestas deciduais e as estratégias de conservação. In Tópicos atuais em botânica. (T.B. Cavalcanti, B.M.T. Walter, G.P. Silva, A.E. Ramos, J.F. Ribeiro, M.C. Silva, R.B. Neves-Alves \& T.A.B. Dias, orgs.) Sociedade Botânica do Brasil/Embrapa Recursos Genéticos e Biotecnologia, Brasília, p.183-188.

TREJO-TORRES, J.C. \& ACKERMAN, J.D. 2002. Composition patterns of Caribbean limestone forests: are parsimony, classification, and ordination analyses congruent? Biotropica 34:502-515.

VIANA, V.M. \& TABANEZ, A.A.J. 1996. Biology and conservation of forest fragments in the Brazilian Atlantic Moist Forest. In Forest patches in tropical landscapes (J. Schelas \& R. Greenberg, eds.). Island Press, Washington, p.151-167. 